\title{
Materialidad escolar en instituciones de cuidado y educación para párvulos en la ciudad de México, 1870-1940
}

School Materiality in Institutions of Care and Education for Children under 6 Years of Age in Mexico City, 1870-1940

Materialidade escolar em instituições de cuidado e educação para crianças menores de 6 anos na Cidade do México, 1870-1940

Adriana Alejandra García-Serrano*

(iD) orcid.org/0000-0002-7052-5553

Para citar este artículo

García-Serrano, A. (2021). Materialidad escolar en instituciones de cuidado y educación para párvulos en la ciudad de México, 1870-1940. Revista Colombiana de Educación, 1(83), 1-17. https://doi.org/10.17227/rce.num83-10867.

Fecha de recepción: 31/10/2019

Fecha de evaluación: 03/09/2020

\section{(9) $\underset{0}{(1) \otimes}$}

Maestría en Ciencias en la Especialidad de Investigaciones Educativas (DIE-Cinvestav). Profesora del Departamento de Investigaciones Educativas-Cinvestav, México. Correo electrónico: aagarcias@cinvestav.mx. 


\section{Resumen}

\section{Palabras clave: Historia \\ educacional; educación de la \\ primera infancia; cultura \\ escolar; kindergarten; \\ educación preescolar}

El objetivo de este artículo de investigación es analizar los materiales escolares en las instituciones de cuidado y educación para niños menores de 6 años en la ciudad de México en el periodo 1870-1940. De manera particular se reflexiona en torno a los materiales utilizados para la instrucción o enseñanza de los párvulos, así como sus cambios y el vínculo que guardaron con lo que se esperaba que los niños pequeños aprendieran, fueran e hicieran en un proceso de apropiación e institucionalización de la educación dirigida a niños pequeños. El enfoque teórico-metodológico incluye ramas de la investigación en historia cultural, específicamente historia de la cultura material, de la materialidad escolar y de la infancia. Con este enfoque se pretende identificar las transformaciones de los materiales escolares y sus usos a partir de la indagación en diversas fuentes bibliográficas y documentos de archivo como inventarios, programas, solicitudes de materiales y recursos, entre otros. Se muestra cómo es que los materiales escolares fueron cambiando a la par que se transitaba de una concepción instructiva a una educativa en las instituciones para niños menores de 6 años.

\section{Abstract}

The aim of this research article is to analyze school materials in care and education institutions for children under 6 years of age in Mexico City in the period 1870-1940. It reflects on the materials used for the instruction or teaching of preschool children; as well as their changes and the link they kept with what young children were expected to learn, go, and do in a process of appropriation and institutionalization of education aimed at young children. The theoreticalmethodological approach includes branches of research in cultural history, specifically the history of material culture, school materiality and childhood. This approach is intended to identify the transformations of school materials and their uses based on research in various bibliographical sources and archival documents such as inventories, programs, requests for materials and resources, among others. It shows how the school materials were changing while moving from an instructive to an educational conception in institutions for children under 6 years of age.

\section{Resumo}

0 objetivo deste artigo de pesquisa, é analisar materiais escolares em instituições de acolhimento e educação para crianças menores de 6 anos de idade, na Cidade do México no período de 1870 a 1940. Em particular, reflete-se sobre os materiais utilizados para a instrução ou ensino de crianças em idade pré-escolar; bem como suas mudanças e o vínculo que mantinham com o que se esperava que as crianças aprendessem, fossem e fizessem, em um processo de apropriação e institucionalização da educação voltada para crianças de pré-escolar. A abordagem teórico-metodológica inclui ramos de pesquisa em história cultural, especificamente a história da cultura material, materialidade escolar e infância. Essa abordagem visa identificar as transformações dos materiais escolares e seus usos a partir da investigação em várias fontes bibliográficas e documentos de arquivo como inventários, programas, solicitações de materiais e recursos, entre outros. Finalmente, mostra-se como os materiais escolares estavam mudando ao passar de uma concepção instrutiva para uma educacional em instituições para crianças menores de 6 anos. 


\section{Introducción}

Este trabajo reflexiona en torno a los materiales escolares de instituciones encargadas del cuidado y educación de niños menores de 6 años en una época en que dichos establecimientos estaban en proceso de definición en la ciudad de México: no existía una uniformidad de concepto, ni una denominación común para nombrarlos, ni tampoco un rango etario determinado para ellos. Aproximarnos a las distintas escuelas para la pequeña infancia implica distanciarnos de una historia continua y ordenada, ya que su creación no fue un proceso en el que una sustituyó a otra; al contrario, se identifican traslapes, de manera que reconocerlas como instituciones diferenciadas puede ser impreciso. En consecuencia, precisaré a qué tipo de establecimientos se refiere este artículo.

La historia de las escuelas para la niñez pequeña en México es posible rastrearla cuando menos desde el siglo XVIII, Josefina Granja (1998) da cuenta de la existencia de las Amigas o "Migas", es decir, establecimientos para niñas en los que además se admitían niños pequeños, desde 1746, época en la que fueron casi la única opción de educación para las niñas. Brindaban cuidado y enseñanza religiosa a los niños más pequeños; sin embargo, con el paso del tiempo su función instructora se fortaleció. Desde finales del siglo XVIII, aceptaban a niños de 2 o 3 años (Tanck, 1984), en el último tercio del siglo XIX atendían a niños a partir de 3 años hasta adultos de 30; su organización era muy semejante a la de las primarias y su financiamiento provenía de los ayuntamientos, aunque las había también privadas. Élida Campos Alba (2013) menciona que en estos años existían Amigas de ambos sexos o solo de niños también. De acuerdo con Mílada Bazant (2012), para la década de 1890 ya no existían Amigas en la ciudad de México.

En la década de 1880, existía una diversidad de establecimientos que incluían a niños menores de 6 años. Entre ellos se encontraban las escuelas elementales de enseñanza, que fueron producto de una reestructuración emprendida durante el gobierno de Manuel González, cuyo fin era constituir una escuela previa a la primaria para niños de 5 a 8 años. Hacia 1888, estas escuelas fueron suprimidas como parte de la reorganización en materia educativa.

A partir de 1883, a las escuelas elementales creadas por Manuel González se les comenzó a llamar escuelas de párvulos, cuyo objetivo era "proteger a los pequeños de las clases menos favorecidas de permanecer solos en sus domicilios por largas horas mientras sus padres salían a trabajar" (Campos, 2013, pp. 128-129). En ellas comenzó a utilizarse el método froebeliano y fueron consideradas por Estefanía Castañeda ${ }^{1}$ (1980) como antecedente del Kindergarten. Entre 1880 y 1886, se crearon cuatro escuelas de párvulos en la ciudad de México sostenidas por el Ayuntamiento. De acuerdo con Campos (2013), formaban parte de la instrucción

1 Estefanía Castañeda (1872-1937), maestra normalista originaria del estado de Tamaulipas, donde se inició como maestra y participó como representante en el Congreso Pedagógico de Tamaulipas en 1899 (Cano, 2000, p. 228). Fue fundadora de los primeros Kindergarten en la capital del país, ocupó diversos cargos como directora del Kindergarten "Froebel" e inspectora. También fue autora de libros escolares y artículos pedagógicos, dirigió el periódico Kindergarten e intervino en diversos congresos internacionales.

Número 83, Tercer cuatrimestre de 2021 (septiembre-diciembre) || e-ISSN: 2323-0134 || 3 
primaria, aunque se ubicaran en edificios separados de esta, lo que apunta hacia una educación de párvulos gestada en articulación a la concepción y normatividad de la educación primaria. En 1892, dichos establecimientos se convirtieron en escuelas primarias para niñas con una sección anexa para párvulos.

Entre 1903 y 1906, se fundaron cuatro escuelas de párvulos a las que en 1907 se les Ilamaría oficialmente Kindergarten. El proyecto del Kindergarten en México surgió de y como una reorganización de las escuelas de párvulos para recibir a niños de 4 a 6 años. Fue publicitado como un "sistema pedagógico de vanguardia" (Cano, 2000, p. 224) que educaría a los niños para el progreso del país. Fueron descritos como la extensión del hogar y las maestras como madres sustitutas. En sus inicios, predominó la doctrina froebeliana y el juego como elemento educador de los niños.

La denominación jardines de niños se generaliza en sustitución de Kindergarten en el periodo posrevolucionario (1920-1940). Para estas instituciones, se acuerda tomar lo mejor de las propuestas de Froebel y Montessori adaptadas a la realidad mexicana, además de incorporarse la pedagogía activa representada por John Dewey. En la década de 1920, los jardines de niños fueron estrechamente vinculados con una educación preparatoria para la primaria, aunque se les continuó considerando como reproductores de un ambiente hogareño; comienza su expansión por el territorio nacional y su consolidación institucional mediante la regulación y control por parte del Estado. Hacia la década de los 30, a esta función educativa se le sumó una concepción de asistencia social que ofrecía el bienestar necesario para el desenvolvimiento apropiado de los niños menores de 6 años. En 1941, el presidente Manuel Ávila Camacho creó el Departamento de Educación Preescolar dependiente de la Secretaría de Educación Pública (SEP), con lo que se subscribió la especificidad educativa de una institución que había surgido casi como parte de la escuela primaria.

\section{Metodología}

Este trabajo está pensado desde el enfoque de la historia cultural de la educación, específicamente en el ámbito de los estudios sobre materialidad y cultura escolar, así como historia de la infancia. La cultura escolar ha sido abordada por diversos autores, entre ellos Dominique Julia quien la define como

un conjunto de normas que definen los saberes a enseñar y las conductas a inculcar, y un conjunto de prácticas que permiten la transmisión de estos saberes y la incorporación de estos comportamientos; normas y prácticas subordinadas a unas finalidades que pueden variar según las épocas. (1995, p. 131)

Desde la perspectiva de Agustín Escolano (2000), la cultura escolar es delimitada como un cúmulo de normas, teorías y prácticas que organizan la regulación de los sistemas, lenguajes y acciones en las escuelas. A ello se suma el reconocimiento de la polisemia del término y la existencia de una pluralidad de culturas escolares (Viñao, 1996), es decir, este tipo de cultura no es constante ni igual en todas las instituciones educativas (Rockwell, 2007; 2016).

Por otra parte, la materialidad escolar es concebida por Viñao como el "entorno físicomaterial y objetos" (2002, p. 75) de las instituciones. Élida Campos (2013) especifica que dicha materialidad consiste en los edificios, espacios no construidos, moblaje, material didáctico y útiles escolares. Para Carlos Ortega (2015), sus significados derivan de los 
propósitos que social y culturalmente se atribuyan a la enseñanza en cada época histórica. Además, esta materialidad no es neutra, es decir, conlleva objetivos políticos e ideológicos (Chaoul, 2019). Desde un enfoque sociocultural, Gabriela Naranjo (2011) entiende la materialidad del aula como "un complejo de signos que juegan un papel fundamental en la transmisión de cultura escolar" (2011, p. 3), donde el sentido y usos de esa materialidad son transformados por maestros y alumnos en función de sus propios intereses y metas. Para Eugenia Roldán (2015), el seguimiento de escuelas específicas permite identificar los cambios y continuidades de su materialidad escolar; a lo que Viñao (2002) agrega la comprensión de cómo se genera el cambio educativo en las instituciones escolares.

Para este trabajo se considera a la materialidad de la escuela como el conjunto de objetos, materiales, tiempos y espacios-lugares adaptados o creados en un tiempo y lugar específicos para el cuidado y educación de los niños menores de 6 años en instituciones o escuelas: edificio escolar, horarios, mobiliario, materiales escolares, canciones, música, juguetes, libros y cuentos, así como a las relaciones y experiencias establecidas entre los objetos, autoridades educativas, maestras y niños. Se reconoce que la materialidad no es neutral, conlleva valoraciones sociales e intereses individuales y colectivos.

Desde la historia de la infancia, Campos (2013) afirma que el pensamiento social en torno a ella se forjó en México a finales del siglo xviıI. Por otra parte, Beatriz Alcubierre (2017), apoyándose en Ariès, reconoce la relatividad del concepto de infancia y lo identifica además como un término flexible y elástico. Esta autora afirma que la noción moderna de infancia comenzó a delinearse en el siglo XVII "a través de una red de dispositivos discursivos e institucionales cuya expresión práctica fue el control obsesivo del cuerpo, la mente y el espacio de los niños por parte de los adultos" (p. 323). Así, retomando a Ariès y Benjamin, define a la infancia como "una construcción de la modernidad definida por la atribución de ciertas características, mediante la intervención de una serie de instituciones" (p. 324), la infancia en la modernidad es también un conjunto de prácticas que regulan la relación adulto-niño.

Las preguntas que guían el análisis en este trabajo son las siguientes: ¿cómo fueron cambiando los materiales escolares para niños pequeños con el tiempo?, ¿cómo existieron ahí y qué pasó con ellos? (Lawn, 2005), ¿qué nos dicen estos objetos de las maneras de instruir y educar a la infancia pequeña?, ¿por qué fue importante crear objetos y utilizar materiales específicos para la educación de los párvulos?

La paulatina construcción de una materialidad escolar solo puede identificarse en la larga duración, desde la que es posible apreciar irrupciones, cambios y permanencias de lo que se esperaba que aprendieran, fueran e hicieran los niños, en este caso los menores de 6 años. En este sentido, Elsie Rockwell sostiene que la dinámica propia que poseen las culturas escolares genera procesos que "no respetan los tiempos económicos y políticos" (2007, p. 178). Los usos de los objetos escolares están enmarcados en reglas de práctica y significados específicos, su análisis requiere de una temporalidad a largo plazo debido a que con el tiempo desarrollan nuevos significados para nuevos propósitos escolares.

Por otra parte, Martin Lawn (2005) establece una relación entre los objetos existentes en los salones de clase y el currículo escolar. Así, la transformación de las ideas pedagógicas y los contenidos a enseñar conllevan necesariamente al cambio de la materialidad de la escuela. De acuerdo con Roldán (2015), a finales del siglo xIx tuvo lugar el debate respecto al término 
educación en diversos países de Hispanoamérica y Francia, que en este periodo comenzó a adquirir el significado de desarrollo de todas las facultades del individuo (intelectual, moral y física) frente a la noción de instrucción, relacionada con la transmisión de conocimiento, así como la transformación del lenguaje en lo educativo. El Tratado elemental de pedagogía escrito por Manuel Flores define a la educación como el "arte que tiende al desarrollo de nuestras facultades, favorece indirecta pero eficazmente el correcto desempeño de nuestros actos, y por consiguiente, hace posible la satisfaccion de nuestras necesidades tanto físicas como intelectuales y morales" (1887, p. 21), la educación atiende el desarrollo de todas las facultades de la persona de forma artificial y deliberada (p. 11).

En el mismo sentido, en un discurso pronunciado en 1902 al instaurar el Consejo Superior de Educación, Justo Sierra insistía en sustituir la instrucción por educación, ya que una instrucción sin educación "es vana y dañosa" (citado en Meneses, 1998, p. 598). Meneses señala que es a principios del siglo xx, que la educación adquirió su característica de integral, entendida como aquella que va "dirigida al hombre entero: cuerpo y mente, capacidades físicas, cognoscitivas, afectivas y volitivas" (1998, p. 777). Esta perspectiva nos ayuda a comprender las diferencias entre la materialidad escolar de las Amigas, escuelas de párvulos, Kindergarten y jardines de niños, y cómo esa materialidad fue producto de a la vez que incidió en la transición de la noción de instrucción a la de educación en dichas instituciones.

\section{Resultados}

\section{Las escuelas Amigas: espacios de cuidado e instrucción para niños pequeños}

Las escuelas Amigas eran diversas tanto en la población que atendían como en lo que enseñaban. La heterogeneidad de estas escuelas era reflejo de la situación misma del país; México era un "territorio de contrastes" (Bazant, 1993, p. 16). La denominación escuela Amiga se aplicaba a establecimientos para niños, adolescentes, adultos e incluso nocturnos.

Se sabe que las condiciones de las escuelas Amigas eran precarias, ocupaban en ocasiones un solo salón o dos. Sus inventarios (Archivo Histórico del Distrito Federal [AHDF], Fondo Ayuntamiento y Gobierno del Distrito, Sección o serie: Instrucción Pública en general, vol. 2495, exp. 1857, fojas 1-2, 1885. AHDF, Fondo Ayuntamiento y Gobierno del Distrito, Sección o serie: Instrucción Pública en general vol. 2495, exp. 1904, fojas 1-3, 1885) no solo refieren a los muebles y útiles que allí existieron, incluyen también la cantidad y en algunos casos sus medidas (en cuartas o varas), color y estado. En dichos documentos, se observan coincidencias en el tipo de mobiliario, por lo general incluyen: mesas, bancos o sillas, pizarrones, caballetes y tinteros. Se deduce que los alumnos compartían mesas, además, en ocasiones se hace referencia a banquillos individuales o bancas en las que cabían varios niños; por ejemplo, un establecimiento reportó tener en 1878 "2 banquillos muy usados" (AHDF, Fondo Ayuntamiento y Gobierno del Distrito, Sección o serie: Instrucción Pública e inventarios, vol. 2665, exp. 1, foja 3, 1879).

Las Amigas contaban por lo menos con un pizarrón, había quienes tenían hasta 5 entre grandes y chicos, aunque no todos se hallaban en buen estado. Entre el mobiliario de estas 
escuelas figuraron carteles de madera y manta, muestras de escritura, mapas de geografía y de la República Mexicana (también llamados "carta mural"), rótulos de lienzo, restiradores, reglas, escuadras, pizarras, tripié y tarimas para los guías. Los materiales como gises y tinta eran adquiridos con personas que los ofrecían de manera particular; muestra de ello fue la solicitud del señor Luis Ortiz a la Comisión de Instrucción en 1885 para que se le compraran a 2 reales el ciento de gises y a un real y cuartilla el cuartillero de tinta asegurando su buena calidad (AHDF, Fondo Ayuntamiento y Gobierno del Distrito, Sección o serie: Instrucción Pública en general, vol. 2495, exp. 1904, fojas 1-3, 1885).

En las Amigas los libros de texto eran escasos y en ellos raramente se encontraban ilustraciones, por lo que una práctica común era que los niños compartieran un mismo libro (Rockwell y Roldán, 2010). En los documentos consultados, solo una Amiga los incluyó en su inventario: "Ocho libros segundos. Cinco id terceros. Veintitres id cuartos. Cuatro aritméticas. Veinte gramáticas. Veinte libros de historia. Cuatro id de geografía. Dos id de geometría. Un libro titulado 'Yns cripum de niñas'" (AHDF, Fondo Ayuntamiento y Gobierno del Distrito, Sección o serie: Instrucción Pública en general, vol. 2495, exp. 1857, fojas 1-2, 1885), la directora especifica que hay más libros que no se incluyen en el inventario debido a que están prestados entre las alumnas.

Es probable que los materiales en las Amigas no fueran suficientes para todos los alumnos, a lo que se sumaba su desgaste debido al uso cotidiano. En este sentido, en la escuela del director José $M$. Barrios había 2 bancos para los niños "cuyas condiciones eran inútiles para su uso", una mesa para el director "inutilísima", 4 restiradores inútiles y de los 14 tinteros de plomo 6 estaban inservibles (AHDF, Fondo Ayuntamiento y Gobierno del Distrito, Sección o serie: Instrucción Pública e inventarios, vol. 2665, exp. 1, foja 17, 1879); sin embargo, también se enlistan materiales calificados como "en buen uso".

De acuerdo con los inventarios de dichos establecimientos, es posible inferir que el juego no era una actividad central para los niños que asistían a ellos (lo que no significa que no jugaran). Los objetos enlistados sugieren prácticas relacionadas con lo escolarizado en semejanza con la primaria y no con un uso lúdico, aun para los niños más pequeños que eran pensados como tabula rasa. Finalmente, los muebles y útiles de los que disponían las Amigas son indicio de que la atención hacia los niños menores de seis años no era solamente de cuidado, sino instructiva también.

\section{Las escuelas de párvulos y la necesidad de una materialidad concreta para la pequeña infancia}

Las escuelas de párvulos mexicanas han sido poco investigadas, sin embargo, es posible aproximarnos a su materialidad y prácticas mediante lo que Josefina Granja (1998) denominó literatura gris, refiriéndose a documentos elaborados con el fin de organizar y regular tanto el funcionamiento como los recursos de las escuelas. Para este trabajo, la literatura gris incluye inventarios, programas, normatividad, etc. Como mencioné anteriormente, entre 1880 y 1886 se establecen 4 escuelas de este tipo en la ciudad de México (AHDF, Fondo Ayuntamiento y Gobierno del Distrito, Sección o serie: Instrucción Pública en general, vol. 2496, legajo 22, exp. 2033, foja 10, 1886; Campos, 2013) en casas arrendadas que se tenían que adaptar y reparar para ser mínimamente apropiadas al fin destinado. Respecto a la apertura del primer 
establecimiento de este tipo, la maestra Estefanía Castañeda enfatizó: "iPero con cuántas dificultades, cuántos esfuerzos para fundar la primera escuela de párvulos en la ciudad de México!" (1980, p. 29). El mobiliario para esta institución se hizo con pocos recursos, un modesto carpintero mexicano fabricó los dones de Froebel imitando el procedimiento de Laubscher ${ }^{2}$ indicio de una representación de los niños a la cual se le reconocían ciertas capacidades físicas, intelectuales y morales específicas.

Por su parte, Manuel Cervantes Ímaz estableció en el año de 1884 una sala de párvulos dentro de la escuela que él dirigía, lo que lo convirtió en maestro pionero de estas escuelas. Cervantes retomó las doctrinas de Pestalozzi y Froebel delineando una educación natural y práctica para el niño, basada en el método objetivo que le brindara las nociones más importantes del mundo que lo rodeaba y los conocimientos que más tarde desarrollaría.

En el Primer Congreso de Instrucción Pública (1889-1890), se definieron algunas de las características de las escuelas de párvulos: su duración sería de 2 años, procurarían el desarrollo físico, intelectual y moral de los niños, además estarían a cargo de mujeres (Marín, 2008). Esta noción de "desarrollo" en esos 3 ámbitos coincide con la función "educativa" de la enseñanza, que en ese periodo empieza a ser entendida como el desenvolvimiento integral de todas las facultades de los alumnos, por oposición a la función meramente "instructiva" que consistía únicamente en la transmisión de conocimientos intelectuales.

De acuerdo con Rosalía Meníndez los salones de clases de las escuelas primarias comienzan a mostrar signos de cambio a partir del Primer Congreso Higiénico Pedagógico de 1882 y los Congresos de Instrucción Pública de 1889-1890 y 1890-1891, debido a que se empieza a procurar un "mobiliario higiénico y moderno, así como útiles que no afectaran la salud de los niños" (2008, p. 249). Por su parte, los oficios de solicitudes o autorizaciones para la adquisición de materiales muestran que el mobiliario de las escuelas de párvulos difería respecto al de las Amigas; sin embargo, ciertos muebles como mesas y sillas permanecieron constantes. Uno de los cambios que puede apreciarse es que para los establecimientos de párvulos se contempló la adquisición de adornos (AHDF, Fondo Ayuntamiento y Gobierno del Distrito, Sección o serie: Instrucción Pública en general, vol. 2496, exp. 2018, foja 9, 1885). Esto podría ser indicio de que dichas instituciones pretendían un aspecto distinto al de las Amigas, quizá lúdico o infantilizado.

La necesidad de contar con mobiliario y útiles acordes al tamaño de los niños surgió desde el inicio de estas escuelas: "La Directora de la Escuela de párvulos participa que la niña Virginia Manterola se lastimó un brazo por haber caído de una banca y solicita se surta al establecimiento de los muebles adecuados a la edad de los niños" (AHDF, Fondo Ayuntamiento y Gobierno del Distrito, Sección o serie: Instrucción Pública en general, vol. 2492, exp. 1613, foja 1. 1883). Así, la fabricación y adaptación de muebles y materiales para la enseñanza de la pequeña infancia comenzó a considerarse una necesidad: los párvulos requerían un mobiliario acorde a sus características físicas e intelectuales; indicio de ello era la utilización

2 Enrique Laubscher (1837-1890), maestro de origen alemán. Se trasladó a México, donde trabajó como profesor, fue director de la escuela Esperanza en el estado de Veracruz — dicho establecimiento fue considerado por Castañeda (1980) como el "el origen del kindergarten mexicano" (p. 28) - y de la Escuela Normal en la ciudad de México. Entre sus aportaciones resaltan la implantación del método simultáneo, la anulación de la instrucción memorística y la promoción del Kindergarten, entre otros. 
de los dones de Froebel. En este mismo sentido, en el programa para dichas escuelas establecido en un reglamento de 1885 (AHDF, Fondo Ayuntamiento y Gobierno del Distrito, Sección o serie: Instrucción pública en general, vol. 2496, exp. 2000, fojas 22-23, 1885) se mencionan, además de los dones froebelianos, materiales como estampas, tejidos y pequeñas canciones.

Por otro lado, las escuelas de párvulos contaban con recursos limitados, las fuentes dan cuenta de la insuficiencia de material y la demora de la Comisión de Instrucción para atender las peticiones de útiles de las maestras (AHDF, Fondo Ayuntamiento y Gobierno del Distrito, Sección o serie: Instrucción Pública en general, vol. 2492, exp. 1614, fojas 1-2, 1883). Era común que estas solicitaran recursos para el funcionamiento de dichas instituciones, en 1885 la directora de una escuela de párvulos pidió al presidente de la república "auxiliar con 12 centavos diarios por cada alumno que se inscriba en las escuelas de párvulos" (AHDF, Fondo Ayuntamiento y Gobierno del Distrito, Sección o serie: Instrucción Pública en general, vol. 2496, exp. 2052, foja 1, 1885).

La idea del niño pequeño como sujeto de enseñanza comienza a ser más visible en este periodo. La educación era el medio para que los niños desarrollaran su iniciativa, personalidad, simpatía, solidaridad, fueran creativos, piadosos y activos; "la escuela instruiría a los párvulos para alcanzar el progreso como individuos y parte de la sociedad" (García y Roldán, 2019, p. 60 ), en otras palabras, se trataba de formar al futuro ciudadano. Si la casa no ofrecía los medios y ambiente adecuados para ello, el Estado establecería escuelas donde los niños pudieran crecer en una atmósfera creada para ellos que, además sería semejante al hogar.

\section{El Kindergarten: institución ad hoc para niños menores de 6 años}

Estas instituciones surgieron como una reorganización de las escuelas de párvulos de finales del siglo XIX, criticadas por intelectuales de la época que señalaron entre otras dificultades edificios escolares inadecuados - salones en mal estado, hacinamiento de alumnos, falta de espacio para recreos, etcétera- y el empleo de los dones froebelianos de manera monótona. Por el contrario, se planteó al Kindergarten como una institución distinta de las escuelas de párvulos respecto a su organización, programas, métodos disciplinarios y edificio escolar. Se le consideró una escuela-hogar, a la vanguardia educativa y una institución ad hoc para el niño pequeño.

Las listas de materiales de los Kindergarten reflejan un cambio significativo respecto a las Amigas y escuelas de párvulos. Se pensó que el niño no debía realizar actividades consideradas escolares como el aprendizaje formal de la lectura, escritura y aritmética con ejercicios de repetición que lo obligaran a permanecer sentado largo tiempo; actividades consideradas desvinculadas de las necesidades de la vida del niño (Castañeda, 1980). Esto no significó la salida de la lectura, escritura y aritmética de los programas, pero se enseñarían sus elementos básicos desde un enfoque distinto: se fomentaría el lenguaje por medio de cuentos, conversaciones, aprendizaje de letras de canciones sencillas, rimas, comparación de elementos fónicos entre sonidos de animales y la voz humana; los niños incrementarían su vocabulario por medio del aprendizaje de los nombres de los objetos que le rodeaban y de su nombre propio completo, entre otras actividades. La enseñanza de la aritmética se sustituyó por nociones de número, medidas, fracciones como mitades y cuartos, así como series numéricas hasta el 4 y combinaciones hasta el 8 por medio de los dones froebelianos. 
En los Kindergarten al igual que en las escuelas de párvulos, el tamaño de los muebles continuó representando una dificultad. Su adquisición no distó significativamente respecto a las escuelas de párvulos, dotarlas de moblaje adecuado fue un proceso lento en el que había que sortear varios obstáculos, entre ellos la escasez de recursos. El mobiliario para los Kindergarten se construyó a medida por fabricantes nacionales, un ejemplo fue la petición que en 1917 se le hizo a la Escuela de Arte Industrial Vasco de Quiroga para fabricar sillas, estantes y mesas destinadas a dichas instituciones de la capital (AHDF, Fondo Ayuntamiento y Gobierno del Distrito, Sección o serie Gobierno del Distrito: instrucción pública, vol. 1659, exp. 6, fojas $1-8,1917)$, aunque también se realizaron compras en el extranjero y adaptaciones.

En el proyecto del Kindergarten, los párvulos debían desarrollar su naturaleza psicofísica jugando, en la naturaleza, con una libertad ordenada, en movimiento, en una atmósfera semejante a la del hogar, armónica y con el amor de su maestra. Para ello los espacios, muebles y útiles tenían que ser acordes a estos preceptos. Estefanía Castañeda afirmó: "El mobiliario, material, útiles y juguetes del niño, así como el edificio, se sujetarán al modelo de un hogar bien constituido" (1980, p. 101). Al menos en las prescripciones oficiales, se comienza a prestar atención a la higiene de estos establecimientos y a la vigilancia del material escolar a través de la función inspectora encomendada a Estefanía Castañeda en 1907 (Acuerdo referente á la inspección de las escuelas nacionales de párvulos, BIPBA, tomo VII, no. 1-3, 1907, pp. 602-605). Además, el diseño y construcción de moblaje comenzó a atender pautas antropométricas e higienistas.

Bajo la idea de poner a los niños en contacto con el medio que les rodea y con la naturaleza (Castañeda, 1980), en los primeros programas (Proyecto de Escuela de Párvulos presentado á la Subsecretaría de Instrucción Pública, Estefanía Castañeda, Boletín de Justicia e Instrucción Pública [en adelante BJIP], tomo II, núm. 2, 1903, pp. 65-90. Programa de la Escuela de Párvulos núm. 2, formado por el director de la misma, Rosaura Zapata, Boletín de Instrucción Pública y Bellas Artes [en adelante BIPBA], tomo v, núm. 6-8, 1906, pp. 685-703) se identifica el uso de materiales caracterizados por su diversidad por medio de los cuales los niños podrían aprender sus características y propiedades al manipularlos, observarlos y dibujarlos: flores, conchas, caracoles, semillas, arena, barro, zacate, palma, diferentes tipos de papel y tela, tijeras, pegamento, pinturas, entre otros.

Los juguetes fueron incluidos en estos programas como objetos que contribuían al desarrollo físico de los pequeños: pelotas, raquetas, cuerdas, canicas, trompos, papalotes, muñecos y "diversos juguetes pertenecientes á los niños" (Proyecto de Escuela de Párvulos presentado á la Subsecretaría de Instrucción Pública, Estefanía Castañeda, BJIP, tomo II, núm. 2,1903, p. 70). Con el tiempo e influencia de los Kindergarten estadounidenses se acepta la transformación de los dones froebelianos, para ello se compró mobiliario, material y libros de consulta por medio de la Librería Purón e Hijos, establecida en la ciudad de México (Zapata, 1951).

Los silabarios y libros de historia, geografía y aritmética desaparecieron de los Kindergarten; en su lugar, se impulsó el uso diario de cuentos y libros de estampas de paisajes, flores, frutos, entre otros (Proyecto de Escuela de Párvulos presentado á la Subsecretaría de Instrucción Pública, Estefanía Castañeda, BJIP, tomo II, núm. 2, 1903, p. 70). La manera de relacionarse con estos objetos también se modificó, pues los párvulos los utilizaban para observar elementos de la naturaleza. En el Proyecto de la Escuela de Párvulos 
de 1903 , se menciona que los niños tendrían acceso a ellos durante el recreo, lo que podría haber significado el comienzo de un uso lúdico de los libros en la educación de los niños pequeños. No era frecuente que los cuentos estuvieran ilustrados - situación que coincide con los libros de texto de las escuelas Amigas-, por lo que correspondía a la educadora realizar bosquejos sencillos en el pizarrón o bien mostrarles estampas a los niños que se relacionaran con la narración. En el programa de $Z^{2} a a^{2}{ }^{3}{ }^{3}$ los cuentos tuvieron una presencia importante también, enlistados y organizados por estaciones del año. Por ejemplo, en invierno a los niños se les leería "El nido de mamá Gorriona", "María y el borrego", "La familia de ratones" (Programa de la Escuela de Párvulos núm. 2, formado por el director de la misma, Rosaura Zapata, BIPBA, tomo v, núm. 6-8, 1906, p. 701).

La música en los Kindergarten fue un elemento muy importante con el cual se intentó diferenciarlos de las escuelas antiguas. La señora Refugio Barragán escribió los primeros juegos y compuso las primeras canciones para estas instituciones; desde la perspectiva de Castañeda estos esfuerzos iniciales contribuyeron a romper con la "quietud obligatoria" (1980, p. 31) que se procuraba en las viejas escuelas. A través de la composición de canciones para niños pequeños se procuró reflejar el sentir de la infancia; además, se adoptaron y adaptaron los juegos y canciones de la obra Cantos de la madre, publicada por Froebel en 1844 para fomentar el gusto estético musical en los niños, su inclinación por el trabajo y el bien de la especie humana, lo que constituyó "una reflexión de la armonía de la vida universal" (p. 47).

Castañeda sugirió que los niños escucharan música de instrumentos, cantaran canciones sencillas, hicieran actividad física acompañada de música y se promoviera el reconocimiento de notas musicales; también se utilizaría para evitar el fastidio de los párvulos. Además, la música tendría un fin placentero y ayudaría a mantener la disciplina (Proyecto de Escuela de Párvulos presentado á la Subsecretaría de Instrucción Pública, Estefanía Castañeda, BJIP, tomo II, núm. 2, 1903, pp. 71-72 y 76). Por su parte, Rosaura Zapata incluyó cantos en su programa organizados por temática y agrupados en las estaciones del año. Así, en verano, y específicamente en septiembre, se entonaría el "Himno á la Patria" y "La bandera" (Programa de la Escuela de Párvulos núm. 2, formado por el director de la misma, Rosaura Zapata, BIPBA, tomo v, núm. 6-8, 1906, p. 700); con lo que además se buscaba inculcar en los niños nociones de historia y su formación ciudadana. En el Programa del Jardín de Niños de 1934 las canciones sencillas servirían para que los pequeños adquirieran una correcta expresión oral (Documento Número 6. Programa del Jardín de Niños, Memoria de la Secretaría de Educación Pública [en adelante MSEP], tomo II, 1934, p. 330).

Considerar a la música como fuente de placer, elemento educativo y disciplinario, así como herramienta para desarrollar el gusto estético desde los diversos programas, sugiere que se concebía que el niño era educable a través de ella, además de relacionarla con la alegría y una atmósfera agradable que debía permear en el Kindergarten.

3 Programa elaborado por Rosaura Zapata en 1906, cuando era directora de la Escuela de Párvulos núm. 2. En este documento, se propuso una detallada organización de temas por semana y mes que partían de una idea globalizadora como base de todos los trabajos, además, los contenidos estuvieron organizados en "centros de interés". Incluyó también 9 dones de Froebel, 4 ocupaciones escolares, juegos, cantos y cuentos. El objetivo de estas actividades era desarrollar en el niño habilidades manuales, cognitivas y físicas. 
Con estas instituciones se consolidó la representación del niño pequeño como un ser en desarrollo; correspondía al Kindergarten educarlo en un ambiente que reprodujera el del hogar y fomentara el desenvolvimiento de sus facultades específicas. Es decir, estas instituciones adquirieron una función educativa en el sentido moderno del término.

\section{El jardín de niños: nacionalización institucional y material}

La etapa de consolidación de los jardines de niños coincide con la creación de la Secretaría de Educación Pública (SEP), promulgada el 29 de septiembre de 1921, con José Vasconcelos como su titular. La SEP fue un ministerio de educación con una jurisdicción mucho más amplia y mayores atribuciones que sus antecesores, algo que fue permitido por la ruptura social y política que implicó la revolución armada de 1910-ca. 1920. Entre los cambios más relevantes introducidos a partir de la existencia de esta secretaría de Estado se encuentra la utilización del concepto educación en lugar de instrucción en el propio nombre de la institución. La distinción entre estas nociones se formuló a finales del siglo XIX. Siguiendo a Solana et al. (1981), instruir significó únicamente "vaciar" información en los alumnos, mientras que por educar se entendió el fomento de virtudes y desaliento de vicios, es decir, desarrollar todas las facultades del individuo -físicas, morales, mentales- y no únicamente las intelectuales.

El tamaño de los muebles pareció estar resuelto en los jardines de niños, pues no se ha encontrado evidencia de lo contrario. En sus listas de materiales se observan coincidencias respecto a los existentes en los Kindergarten y se aprecia la introducción de otros como la cera de campeche, hojas de colores, gises, plumas, sobres, gomas, estambre, escobas, escobetas, plumeros y varias clases de papel (AHDF, Fondo Ayuntamiento y Gobierno del Distrito, Sección o serie: Instrucción Pública jardines de niños, vol. 2563, exp. 2, s/f, 19061917. AHDF, Fondo Ayuntamiento y Gobierno del Distrito, Sección o serie: Instrucción Pública jardines de niños, vol. 2563, exp. 4, s/f, 1911-1917).

Esta diversidad de materiales se observa también en el programa de 1934 (Documento Número 6. Programa del Jardín de Niños, MSEP, tomo II, 1934, pp. 328-333), en el que se incluyó la Escuela de la Acción y se eliminaron los dones froebelianos, lo mismo sucedió con las ocupaciones de Froebel hacia la década de 1940. Sin embargo, la idea de que los niños aprendieran en contacto con la naturaleza tuvo continuidad. En este mismo programa, la narración de cuentos serviría para fomentar la cultura estética del niño, acercarlos al conocimiento de la vida, despertar su alma a las emociones y sentimientos, así como para desarrollar su lenguaje y ampliar su vocabulario.

Los materiales con los que trabajarían los párvulos fungirían como herramientas para introducirlos al mundo y conocer los nombres de los objetos que estaban a su alrededor, sus características, usos y propiedades. De esta manera, el moblaje y útiles para su educación cobraron gran importancia, pues, además de no representarles ningún peligro, por medio de ellos se motivaría su curiosidad, iniciativa y espontaneidad. Esto apunta a que, además de reconocerse en el niño capacidades como obediencia, ternura, gracia, inocencia, entre otras, se le miró como un ser capaz de interactuar con su medio a través de los sentidos. Entonces, para motivar su actividad debía rodeársele de un medio estimulante donde pudiera encauzar su creatividad. Castañeda (1980) describió diversas actividades desarrolladas en el Kindergarten en las que, por medio de la manipulación y juego con los objetos, los niños lograban expresarse y conocer el mundo en que vivían. 
La representación del niño de 3 a 6 años era ya distinta de la del adulto y sobre todo de su madre. De este modo, el niño dejaba de ser un adulto en miniatura y la escuela debía ser una institución que enseñara a vivir la particularidad de esa etapa de la vida. En la década de 1930, se crean juegos y se compone música mexicana para ellos, y se procura un mobiliario específico para estas escuelas elaborado por obreros mexicanos como parte del proyecto de nacionalización de estas instituciones. Es decir, comienza a crearse una cultura escolar específica y nacionalista de los jardines de niños, que traía consigo sedimentaciones de instituciones, prácticas escolares y representaciones de la niñez pequeña anteriores, pero que debido a la influencia del higienismo, el avance de la pedagogía, ciencia y tecnología se estaba transformando.

\section{Conclusiones}

Las maneras en que se pensaba a los establecimientos dedicados al cuidado y enseñanza de los niños menores de 6 años en la ciudad de México fueron cambiando a lo largo del tiempo, así como sus métodos, programas, espacios, moblaje y útiles empleados para tales fines. Pensar en torno a los materiales escolares utilizados en dichas instituciones permite recuperar su historicidad, identificar cambios, permanencias e innovaciones; también, en la medida en que las fuentes lo permiten, distinguir los procesos de apropiación e institucionalización de la educación para niños pequeños.

En las escuelas Amigas y de párvulos es posible identificar que los niños pequeños debían ser instruidos de manera muy semejante a la primaria: bajo una estricta disciplina. Los muebles y útiles escolares de las Amigas apuntan hacia su función instructiva, al niño no se le reconocían capacidades innatas por lo que en la escuela debían adquirir conocimientos únicamente intelectuales establecidos con antelación. En las escuelas de párvulos, la idea del niño menor de 6 años como sujeto de enseñanza comienza a ser más evidente a la par de una concepción sobre su educación fuera del hogar en establecimientos escolares que reprodujeran el ambiente doméstico y estuvieran dirigidos por mujeres.

En los discursos sobre el Kindergarten como en sus prácticas se hacen intentos para distinguir a estas instituciones de las escuelas de párvulos de finales del siglo XIX y de las primarias, aunque en aspectos organizativos se aprecian semejanzas respecto a estas últimas. Con los Kindergarten el cuidado y educación de la niñez pequeña tuvieron lugar en estrecho vínculo con el hogar, se procuró además su desarrollo psicofísico y moral. Para ello, el Estado desplegó una serie de esfuerzos para la creación, adaptación y adquisición de materiales, útiles, música y juguetes adecuados a las necesidades físicas y mentales de los párvulos. Además, se le otorgó mayor importancia y recursos a la adaptación de edificios escolares para, entre otros aspectos, acercarlos al ideal del Kindergarten. En estas instituciones y en los jardines de niños, la infancia menor de 6 años ocupó el lugar central para la creación, adaptación, compra y utilización de moblaje y útiles escolares. Aunado a ello, se fue configurando una estética acorde con esta etapa de la infancia, lo que dio lugar a la infantilización de los espacios para el aprendizaje de los niños más pequeños. 
De esta manera, las transformaciones y continuidades de los espacios, muebles y materiales escolares son indicios de la transición del niño pequeño susceptible de ser instruido -en las Amigas y escuelas de párvulos- a configurarse como sujeto educable física, moral e intelectualmente en una institución ad hoc para él y su educación.

Con los jardines de niños, los párvulos ya no eran pensados como adultos pequeños, por lo que uno de los objetivos de estas instituciones era enseñar a vivir la particularidad de la infancia. Para ello, se crearon juegos, se compuso música mexicana y se procuró un mobiliario y utillaje específico para los niños. Es decir, comienza a crearse una cultura escolar concreta y nacionalista de estas instituciones y para niños de 3 a 6 años. En suma, es posible identificar una paulatina configuración de una cultura escolar para el Kindergarten y jardín de niños con los tres elementos esenciales que, según Dominique Julia (1995), definen a este tipo de cultura: "espacio separado, curso dividido en niveles y un cuerpo profesional específico" ( $p$. 134).

\section{Referencias}

\section{Fuentes primarias}

Archivo Histórico del Distrito Federal (AHDF).

Fondo Ayuntamiento y Gobierno del Distrito, Sección o serie: Instrucción Pública jardines de niños, vol. 2563, exp. 2, 1906-1917; vol. 2563, exp. 4, 1911-1917.

Fondo Ayuntamiento y Gobierno del Distrito, Sección o serie: Gobierno del Distrito: Instrucción Pública, vol. 1659, exp. 6, 1917.

Fondo Ayuntamiento y Gobierno del Distrito, Sección o serie: Instrucción Pública en general, vol. 2496, legajo 22, exp. 2033, 1886; vol. 2495, exp. 1857, 1885; vol. 2495, exp. 1904, 1885; vol. 2496, exp. 2018, 1885; vol. 2492, exp.1613, 1883; vol. 2492, exp. 1614, 1883; vol. 2496, exp. 2052, 1885; vol. 2496, exp. 2000, 1885.

Fondo Ayuntamiento y Gobierno del Distrito, Sección o serie: Instrucción Pública e inventarios, vol. 2665, exp. 1, 1879.

Centro de Investigación y Difusión de Educación Preescolar (CIDEP).

Archivo Histórico de la Secretaría de Educación Pública (AHSEP)/Archivo General de la Nación (AGN).

Acuerdo referente á la inspección de las escuelas nacionales de párvulos. bipba, tomo VII, no. 1-3, 1907.

Documento Número 6. Programa del Jardín de Niños, MSEP, tomo II, 1934.

Programa de la Escuela de Párvulos núm. 2, formado por el director de la misma, Rosaura Zapata, bipba, tomo v, no. 6, 1906.

Proyecto de Escuela de Párvulos presentado á la Subsecretaría de Instrucción Pública, Estefanía Castañeda, BJIP, tomo II, no. 2, 1903. 


\section{Fuentes secundarias}

Alcubierre, B. (2017). Niños de nadie. Usos de la infancia menesterosa en el contexto borbónico. Universidad Autónoma del Estado de Morelos-Bonilla Artigas Editores.

Bazant, M. (1993). Historia de la educación durante el porfiriato. Colmex.

Bazant, M. (2012). La educación moderna, 1867-1911. En P. Gonzalbo y A. Staples (coords.), Historia de la educación en la ciudad de México (pp. 245-327). El Colegio de México (Colmex)-Secretaría de Educación Pública (SEP).

Campos, E. (2013). De la escuela de párvulos a los jardines de niños. Construcción de la cultura escolar en la educación preescolar del Estado de México 1881-1926. El Colegio Mexiquense A. C.

Cano, G. (2000). Género y construcción cultural de las profesiones en el porfiriato: magisterio, medicina, jurisprudencia y odontología. Historia y Grafía, 14, 207-243. academia.edu/4103591/Género_y_construcción_cultural_de_las_profesiones_en_el_ Porfiriato_magisterio_medicina_jurisprudencia_y_odontología

Castañeda, E. (1931/1980). Manuales del Kindergarten en Conección con el Primer Ciclo de la Escuela Primaria. Editorial Jus.

Chaoul, M. (2019). Ciudad, espacios y la cultura material de la escuela primaria en México hacia finales del siglo xIX y principios del XX. Anuario de Historia de la Educación, 20(1), 11-27.

https://academia.edu/49138871/Ciudad_espacios_y_la_cultura_material_de_la_escu ela_primaria_en_México_hacia_finales_del_siglo_XIX_y_principios_del_XX

Escolano, A. (2000). Las culturas escolares del siglo xx. Encuentros y desencuentros. Revista de Educación, (Núm. Extra.), 201-2018. https:// redined.mecd.gob.es/xmlui/bitstream/handle/11162/73415/008200230158.pdf?sequ ence $=1$

Flores, M. (1887). Tratado elemental de pedagogía. Secretaría de Fomento. cdigital.dgb.uanl.mx/la/1080022560/1080022560.PDF

García, A., y Roldán, E. (2019). Los inicios de la escolarización de los niños menores de 6 años en la Ciudad de México, 1870-1940: elementos para pensar la representación moderna del preescolar. Revista Mexicana de Historia de la Educación, 7(13), 49-71. http://www.rmhe.somehide.org/index.php/revista/article/view/175/261

Granja, J. (1998). Formaciones conceptuales en educación. Universidad Iberoamericana.

Julia, D. (1995). La cultura escolar como objeto histórico. En M. Menegus y E. González (coords.), Historia de las universidades modernas en Hispanoamérica. Métodos y fuentes (pp. 131-153). Universidad Nacional Autónoma de México (UNAM)-Centro de Estudios sobre la Universidad (CESU). http://cupdf.com/document/la-cultura-escolarcomo-objeto-historico.html 
Lawn, M. (2005). A Pedagogy for the Public: the place of objects, observation, mechanical production and cupboards. En M. Lawn y I. Grosvenor, Materialities of Schooling. Design, Technology, Objects, Routines (pp. 47-70). Symposium Books.

Marín, A. (2008). El porfiriato y su sistema educativo. En A. Marín, Historia de la Pedagogía en el Porfiriato (pp. 21-43). Innovación Editorial Lagares de México.

Meneses, E. (1998). Tendencias educativas oficiales en México 1821-1911. La problemática de la educación mexicana en el siglo XIX y principios del siglo XX. Universidad Iberoamericana.

Meníndez, R. (2008). Memorias de un salón de clase en la ciudad de México: mobiliario y materiales escolares (1879-1911). Foro de Educación, 10, 245-263. https://www.forodeeducacion.com/ojs/index.php/fde/article/view/159

Naranjo, G. (2011). La construcción social y local del espacio áulico en un grupo de escuela primaria. Revista de Investigación Educativa, 12, 2-27. https://uv.mx/cpue/num12/inves/naranjo-construccion-social.html

Ortega, C. (2015). Historia política de la tecnología: una propuesta metodológica para la historia de la arquitectura escolar (Ciudad de México, 1880-1920). Revista Mexicana de Historia de la Educación, 3(6), 159-180. https://www.rmhe.somehide.org/index.php/revista/article/download/63/73

Rockwell, E. (2007). Huellas del pasado en las culturas escolares. Revista de Antropología Social, 16, 175-212. https://revistas.ucm.es/index.php/RASO/article/view/RASO0707110175A/9144

Rockwell, E. (2016). Culturas escolares. En A. Salmerón, B. Trujillo, A. Rodríguez y M. de la Torre (coords.), Diccionario Iberoamericano de Filosofía de la Educación. Fondo de Cultura Económica https://fondodeculturaeconomica.com/dife/definicion.aspx?I=C\&id=45

(FCE)-UNAM.

Rockwell, E., y Roldán, E. (2010). Nuestros pasos por la escuela. Lo que queda y lo que cambia. Ediciones SM.

Roldán, E. (2015). Bildung/Éducation/Education/Educación. Manuscrito inédito.

Roldán, E. (2015). From Monitorial to Graded Schooling in 19th Century Mexico: Politics and Pedagogy in the Definition of Modern Education. En M. Caruso (ed.), Classroom Struggle. Organizing Elementary School Teaching in the 19th Century (pp. 177-201). Peter Lang Edition.

Solana, F., Cardiel, R. y Bolaños, R. (coords). (1981). Historia de la educación pública en México (1876-1976). Fondo de Cultura Económica (FCE)-SEP.

Tanck, D. (1984). La educación ilustrada 1786-1836. Colmex. 
Viñao, A. (1996). Culturas escolares, reformas e innovaciones: entre la tradición y el cambio. Forum Europeo de Administradores de la Educación. Jornadas estatales (8. a , Murcia), 17-29.

Viñao, A. (2002). Sistemas educativos, culturas escolares y reformas. Continuidades y cambios. Ediciones Morata.

Zapata, R. (1951). La educación Preescolar en México. SEP. 